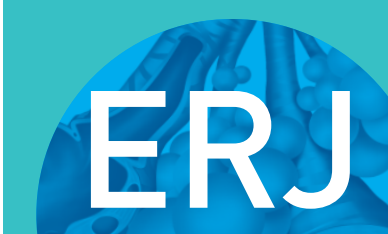

open research

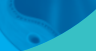

\title{
Donor-derived, cell-free DNA levels by next-generation targeted sequencing are elevated in allograft rejection after lung transplantation
}

\author{
Kiran K. Khush ${ }^{1}$, Iwijn De Vlaminck ${ }^{2}$, Helen Luikart ${ }^{3}$, David J. Ross ${ }^{4}$ and \\ Mark R. Nicolls ${ }^{5}$
}

Affiliations: ${ }^{1}$ Division of Cardiovascular Medicine, Dept of Medicine, Stanford University, Stanford, CA, USA. ${ }^{2}$ Meinig School of Biomedical Engineering, Cornell University, Ithaca, NY, USA. ${ }^{3}$ Division of Cardiovascular Medicine, Dept of Medicine, Stanford University, Stanford, CA USA. ${ }^{4}$ CareDx, Brisbane, CA, USA. ${ }^{5}$ Division of Pulmonary and Critical Care Medicine, Dept of Medicine, Stanford University, Stanford, CA, USA.

Correspondence: Kiran K. Khush, Division of Cardiovascular Medicine, Stanford University School of Medicine, 300 Pasteur Drive, Falk CVRC 263, Stanford, CA 94305, USA. E-mail: Kirandstanford.edu

ABSTRACT Surveillance after lung transplantation is critical to the detection of acute cellular rejection (ACR) and prevention of chronic lung allograft dysfunction (CLAD). Therefore, we measured donorderived cell-free DNA (dd-cfDNA) implementing a clinical-grade, next-generation targeted sequencing assay in 107 plasma samples from 38 unique lung transplantation recipients with diagnostic cohorts classified as: (1) biopsy-confirmed or treated ACR, (2) antibody-mediated rejection (AMR), (3) obstructive CLAD, (4) allograft infection (INFXN) and (5) Stable healthy allografts (STABLE). Our principal findings are as follows: (1) dd-cfDNA level was elevated in ACR (median 0.91\%; interquartile range (IQR): 0.39-2.07\%), CLAD (2.06\%; IQR: $0.57-3.67 \%)$ and an aggregated cohort of rejection encompassing allograft injury (1.06\%; IQR: $0.38-2.51 \%)$, compared with the STABLE cohort (0.38\%; IQR: $0.23-0.87 \%)$ ( $p=0.02)$; (2) dd-cfDNA level with AMR was elevated (1.34\%; IQR: 0.34-2.40\%) compared to STABLE, although it did not reach statistical significance $(p=0.07)$ due to limitations in sample size; (3) there was no difference in dd-cfDNA for allograft INFXN (0.39\%; IQR: $0.18-0.67 \%)$ versus STABLE, which may relate to differences in "tissue injury" with the spectrum of bronchial colonisation versus invasive infection; (4) there was no difference for dd-cfDNA in unilateral versus bilateral lung transplantation; (5) "optimal threshold" for dd-cfDNA for aggregated rejection events representing allograft injury was determined as $0.85 \%$, with sensitivity $=55.6 \%$, specificity $=75.8 \%$, positive predictive value $(\mathrm{PPV})=43.3 \%$ and negative predictive value $(\mathrm{NPV})=83.6 \%$. Measurement of plasma dd-cfDNA may be a clinically useful tool for the assessment of lung allograft health and surveillance for "tissue injury" with a spectrum of rejection.

@ERSpublications

Donor-derived cell-free DNA monitoring in plasma can detect allograft rejection and quiescence after lung transplantation https://bit.ly/3cIQITT

Cite this article as: Khush KK, De Vlaminck I, Luikart H, et al. Donor-derived, cell-free DNA levels by next-generation targeted sequencing are elevated in allograft rejection after lung transplantation. ERJ Open Res 2021; 7: 00462-2020 [https://doi.org/10.1183/23120541.00462-2020]. 


\section{Introduction}

To date, the International Society for Heart and Lung Transplantation (ISHLT) Thoracic Transplant Registry has accrued data on $>69200$ adult lung transplants performed at 260 centres, with $81 \%$ bilateral procedures [1]. Approximately $27 \%$ of recipients experienced at least one episode of treated acute cellular rejection (ACR) during the initial year post-lung transplantation while bronchiolitis obliterans syndrome (BOS), the predominant phenotype of chronic lung allograft dysfunction (CLAD), occurred with an incidence of $\sim 10 \%$ per year post-lung transplantation $[1,2]$. Reported BOS-free survival for bilateral and unilateral lung transplantation recipients remains a sobering 3.16 (95\% CI: 2.99-3.30) and 3.58 (95\% CI: 3.53-3.72) years, respectively [2]. Although CLAD represents the leading cause of mortality for recipients after 1 year post-lung transplantation, allograft non-cytomegalovirus (CMV) infection represents an ongoing threat, representing $20.5 \%$ of mortality events during years 1 to 3 post-lung transplantation [1]. Therefore, novel noninvasive surveillance of allograft health is critical to the early detection of infection or rejection events, in order to mitigate lung transplantation mortality. Furthermore, since CLAD represents a progressive and irreversible type of tissue injury that ultimately culminates in allograft failure, pre-emptive intervention strategies may ultimately improve clinical outcomes.

Donor-derived cell-free DNA (dd-cfDNA) has been extensively investigated as a biomarker of "allograft injury" and has been validated for differentiation of acute cellular rejection (ACR), antibody-mediated rejection (AMR), and quiescence after renal and cardiac transplantation [3-5]. After lung transplantation, AGBOR-ENOH et al. [6,7], while utilising shotgun sequencing methods, described elevated dd-cfDNA levels during ACR and AMR, while the highest tertile dd-cfDNA during the initial 3 months portended a 6.6-fold increased risk for subsequent allograft failure.

In this current study, our aim was to assess the clinical utility of dd-cfDNA measurement implementing a clinical-grade, next-generation targeted (NGT) sequencing assay for the detection of rejection events and lung allograft health, in a biorepository of plasma samples with established clinical-pathological diagnoses after lung transplantation.

\section{Methods}

Histopathology

ACR was graded for trans-bronchial biopsies (TBBx) by an experienced pathologist according to the Revised ISHLT Histopathological Classification as: Grade A (perivascular lymphocytic infiltration) subtypes A0: absence of ACR; A1: minimal; A2: mild; A3: moderate; A4: severe; Grade B (lymphocytic bronchiolitis) subtypes B 0-2R, BX; and Grade C (presence or absence of bronchiolitis obliterans) [8]. AMR was determined by the ISHLT and Banff Lung Pathology working group criteria, which were consistent with "probable" AMR $[9,10]$. BOS was determined by ISHLT consensus council guidelines for classification of CLAD [11, 12].

\section{Biorepository}

The biorepository was derived from the Genome Transplant Dynamics (GTD)([ClinicalTrials.gov identifier: NCT01985412) study at Stanford and included 107 plasma samples with corresponding histopathology by trans-bronchial biopsies (TBBx), bronchoalveolar lavage (BAL) microbiological studies and clinical data from 38 lung transplantation recipients, and classified as: 1) biopsy-confirmed or treated ACR but without biopsy confirmation (ACR); 2) AMR; 3) CLAD; 4) allograft infection (INFXN) without concurrent rejection; and 5) stable healthy allografts (no evidence of rejection or infection) (STABLE). Eligible patients were bilateral or unilateral lung transplantation recipients $\geqslant 15$ years of age, $\geqslant 14$ days post-lung transplantation. Exclusions included: multi-organ transplant, pregnancy or PRBC (packed red blood cells) transfusion within 21 days prior to dd-cfDNA determination.

\section{INFXN category}

Lung allograft INFXN was defined for our data set based on BAL culture isolation for bacterial, fungal or viral pathogens. Neither Procalcitonin nor respiratory viral Luminex ${ }^{\infty}$ PCR tests were performed concurrent with the sample collection era. Chest imaging results were not available for inclusion in this data analysis.

\section{Plasma samples and dd-cfDNA measurements}

Venous blood was collected in Streck ${ }^{\odot}$ Cell-Free DNA BCT tubes prior to performance of bronchoscopy procedures, processed and stored at $-70^{\circ} \mathrm{C}$ for subsequent analysis. Samples were batch analysed in a Central Clinical Laboratories Improvements Act (CLIA) and College of American Pathologists (CAP)-certified laboratory (CareDx, Inc., Brisbane, CA, USA). Details of the standardised specimen processing and analytical methodology for targeted next-generation sequencing (NGS) dd-cfDNA (AlloSure ${ }^{\circledR}$ ) have been previously published $[3,13-15]$. 


\section{Statistical analyses}

Data was assessed for normality and then analysed utilising non-parametric testing with Wilcoxon signed rank test. Values for dd-cfDNA were expressed as median with 25th-75th percentile interquartile range (IQR), 95\% confidence intervals and box plots. The p-values were reported for unique patient samples that were defined as the earliest plasma sample within each group for each patient. The analysis cohorts defined for this study were non-overlapping. The cohort with assigned diagnoses of both ACR and concurrent infection were eliminated from further analysis. Further comparisons were performed for unilateral versus bilateral lung transplantation by Fisher's exact test. Receiver operating characteristic (ROC)-area under the curve (AUC) analysis was performed for the aggregated rejection cohort encompassing allograft injury (i.e. REJXN+AMR+BOS), while positive predictive value (PPV) and negative predictive value (NPV) of rejection were calculated based on ISHLT reported data with prevalence of $25 \%$ for ACR [1]. An "optimal threshold" for dd-cfDNA for the aggregated rejection cohort was determined from this data set as the maximal value for combined sensitivity+specificity.

\section{Results}

\section{Demographics}

Demographics for the lung transplantation patient cohort are depicted in table 1 . The utilised biorepository is depicted in (figure 1) that included 107 plasma samples from 38 unique lung transplantation recipients. TBBx with ACR were graded as follows: Grade A1: 13 samples from eight patients; and Grades A2-A4: nine samples from six patients ( $A 2=5, \mathrm{~A} 3=2, \mathrm{~A} 4=2$ samples). For seven samples from six patients, no histopathological diagnosis was obtained, although patients were treated for ACR with associated clinical improvement. For 59 plasma samples (29 patients) the TBBx were reviewed for concurrent lymphocytic bronchiolitis (ISHLT Grade B), and only one isolated histopathological diagnosis of Grade B1R was identified. Treatment regimens for rejection included: pulsed-dose corticosteroids $(n=27)$, azithromycin $(n=4)$, polyclonal rabbit anti-thymocyte globulin $(n=2)$ and "other" $(n=3)$.

For seven patients there were nine plasma samples associated with HLA donor-specific HLA antibodies (DSA) and classified as "probable" AMR and received treatment with plasmapheresis $(\mathrm{n}=6)$ and rituximab $(\mathrm{n}=3)$. DSA with AMR included: Class I (57\%), Class II (28\%) and combined (14\%). For seven patients there was a clinical-physiological diagnosis of obstructive phenotype CLAD (O-CLAD) or BOS.

\begin{tabular}{|c|c|}
\hline Patients n & 38 \\
\hline Age years mean \pm sD & $53 \pm 17$ \\
\hline Male \% & 63 \\
\hline \multicolumn{2}{|l|}{ Ethnicity \% } \\
\hline White & 80 \\
\hline Black & 8 \\
\hline Asian/Pacific Islander & 8 \\
\hline Hispanic & 4 \\
\hline \multicolumn{2}{|l|}{ Native lung disease $\%$} \\
\hline COPD & 27 \\
\hline IPF & 25 \\
\hline $\mathrm{CF}$ & 24 \\
\hline Bronchiectasis & 10 \\
\hline A1AT & 2 \\
\hline $\mathrm{OB}$ & 2 \\
\hline Sarcoid & 2 \\
\hline Other & 8 \\
\hline \multicolumn{2}{|l|}{ CMV status \% } \\
\hline $\mathrm{D}^{+} / \mathrm{R}^{+}$ & 46 \\
\hline$D^{-} / R^{-}$ & 12 \\
\hline $\mathrm{D}^{+} / \mathrm{R}^{-}$ & 21 \\
\hline $\mathrm{D}^{-} / \mathrm{R}^{+}$ & 21 \\
\hline
\end{tabular}

IPF: idiopathic pulmonary fibrosis; CF: cystic fibrosis; A1AT: $\alpha-1$ anti-trypsin deficiency; OB: obliterative bronchiolitis; CMV: cytomegalovirus; D: donor; R: recipient. 
Respiratory allograft-associated infections, determined by either BAL analysis or ancillary laboratory serological studies, are depicted in table 2 , which included bacterial $(n=22$ patients), fungal ( $n=11$ patients) and viral ( $\mathrm{n}=10$ patients) pathogens. The INFXN cohort included only plasma samples associated with BAL positive culture results in the absence of concurrent allograft rejection.

The dd-cfDNA level was elevated in the spectrum of lung allograft rejection events

As depicted in figure 2, the median dd-cfDNA was significantly elevated in the 29 ACR samples $(0.91 \%$; IQR: $0.39-2.07 \%)$ compared to the 28 samples in the STABLE cohort (0.38\%; IQR: $0.23-0.87 \%$; $\mathrm{p}=0.021)$. No difference was observed for stratifying bilateral versus unilateral lung transplantation recipient groups: 0.46\% (IQR: $0.22-1.06 \%$ ) versus $0.41 \%$ (IQR: $0.20-0.87 \%$ ), respectively (Fisher's test; $\mathrm{p}=0.40$ ). The dd-cfDNA level was also elevated in CLAD (2.06\%; IQR: 0.97-3.34\%) versus the STABLE cohort ( $\mathrm{p}=0.02)$. The cohort of samples with "Probable" AMR had an elevated median dd-cfDNA level of 1.34\% (IQR: $0.34-2.40 \%)$ which was not statistically different compared with the STABLE cohort $(\mathrm{p}=0.07)$. Individual unique lung transplantation patient dd-cfDNA levels in allograft-associated events are depicted in figure 3 for STABLE, ACR, AMR, INFXN and CLAD.

The median dd-cfDNA level for the aggregated cohort that encompassed the spectrum of allograft rejection events with tissue injury (i.e. ACR+AMR+BOS) had a median dd-cfDNA level of $1.06 \%$ (IQR: $0.38-2.51 \%$ ), which was significantly elevated compared with the STABLE cohort $(\mathrm{p}=0.01)$. ROC analysis demonstrated an AUC $=66.7 \%$ (95\% CI: 58.6-73.8\%). An "Optimal Threshold" for dd-cfDNA for aggregated spectrum of rejection was determined as $0.85 \%$ based on the maximal combined sensitivity+specificity $=131.3 \%$. Based on a threshold dd-cfDNA of $0.85 \%$, sensitivity for rejection $=55.6 \%$ (95\% CI: $44.2-66.2 \%)$, specificity $=75.8 \%(95 \%$ CI: 69.3-82.3\%), PPV=43.3\% (95\% CI: 35.6-51.8\%) and NPV=83.6\% (95\% CI: 80.1-87.2\%). Although not a primary focus in this preliminary investigation, figure 4 depicts the different dd-cfDNA levels across distinct clinical events for 19 unique patients with multiple associated samples. Clinical events included: STABLE healthy, ACR, AMR, CLAD, INFXN and follow-up testing 1-2 months after treatment. Individual patient dd-cfDNA levels demonstrated a trend for higher levels in association with AMR and CLAD clinical events.

The dd-cfDNA level was NOT increased in association with lung allograft-associated INFXN or colonisation as defined by BAL microbiological culture results

The dd-cfDNA median value in the 33 allograft-associated INFXN events with absence of concurrent allograft rejection was $0.39 \%$ (IQR: $0.18-0.67 \%$ ), which was not significantly different from the healthy STABLE cohort $(p=0.56)$. Our categorical diagnosis of INFXN was determined by BAL microbiological culture analysis or adjunctive serological testing; however, additional clinical data incorporating chest imaging results were unavailable to permit further detailed distinction of bronchial colonisation versus invasive infection.

\section{Discussion}

In this preliminary study, dd-cfDNA levels by NGT sequencing were significantly elevated in a spectrum of rejection immunological events after unilateral and bilateral lung transplantation. The dd-cfDNA levels

\section{TABLE 2 Bronchoalveolar lavage culture results for bacterial, fungal and viral pathogens in}

\section{the infection cohort}

\section{Bacterial}

Achromobacter xylosoxidans

Pseudomonas aeruginosa

Serratia marcescens

Escherichia coli

Staphylococcus spp.

Stenotrophomonas maltophilia

Klebsiella pneumoniae

\section{Fungal}

Aspergillus spp.

Scedosporium spp.

Viral

Respiratory syncytial virus

Cytomegalovirus

Rhinovirus

22 (51)

2

7

2

Data are presented as $\mathrm{n}(\%)$ or $\mathrm{n}$. 


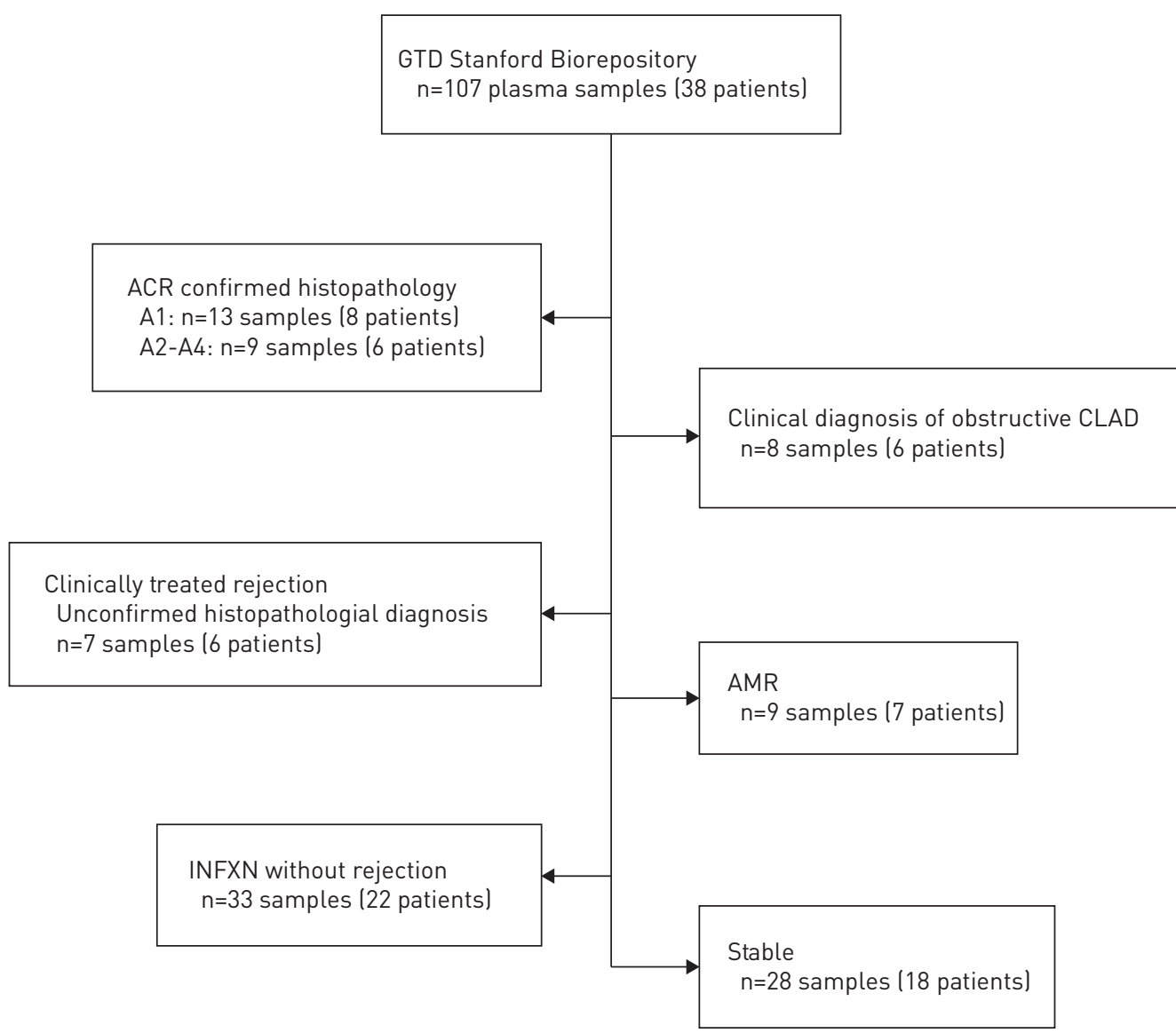

FIGURE 1 Biorepository plasma samples for donor-derived cell-free DNA (dd-cfDNA) analysis and associated diagnostic cohorts in lung transplant recipients. GTD: genome transplant dynamics; ACR: acute cellular rejection; CLAD: chronic lung allograft dysfunction; INFXN: allograft infection in absence of concurrent rejection.

were significantly elevated in both ACR and obstructive phenotype CLAD. The elevated dd-cfDNA level in AMR was also elevated, although did not achieve statistical significance, likely due to limitations in sample size for this cohort. The dd-cfDNA level for the cohort with respiratory allograft INFXN in the absence of concurrent rejection was not different from the STABLE healthy cohort. We speculate that allograft infection represents a continuum from bronchial colonisation to bona fide invasive infection, and this heterogeneity likely explains the lack of allograft "tissue injury" as determined by the plasma dd-cfDNA level. Further prospective investigation should be valuable to correlate allograft injury assessment by dd-cfDNA levels with diverse types and severity of allograft infection.

For the aggregated cohort of rejection events (i.e. ACR+AMR+BOS), the dd-cfDNA median level was $\sim 3$-fold elevated (1.06\%; IQR: $0.38-2.51 \%)$ in contrast to the low level observed in stable healthy patients (0.38\%; IQR: $0.23-0.87 \%)$. An "optimal threshold" for dd-cfDNA for the aggregated rejection cohort encompassing tissue injury was determined from this data as $0.85 \%$ whereupon the sensitivity for the spectrum of rejection $=55.6 \%$, specificity $=75.8 \%, \mathrm{PPV}=43.3 \%$ and $\mathrm{NPV}=83.6 \%$. If further substantiated, we hypothesise that the high NPV should support the utility of this plasma biomarker for allograft surveillance and allow for reduction in invasive diagnostic biopsy procedures. Clinical lung transplantation programmes differ widely regarding protocol biopsies for surveillance of ACR. In a comprehensive report by TRULOck et al. [16], ACR was detected in $\sim 25 \%$ of surveillance FOB procedures, while two-thirds occurred during the initial 6 months after lung transplantation. However, complications of TBBx, in particular bronchial haemorrhage, were significantly more frequent as compared to non-transplant patients. Further, although a sensitivity $>90 \%$ of TBBx for detection of ACR $\geqslant$ ISHLT grade A2 has been reported, $10.8 \%$ of procedures were associated with complications that could limit adequate tissue sampling for histopathological interpretation [17]. Modified FOB techniques have recently been implemented that enhance the adequacy of lung tissue specimens in $96.6 \%$ of procedures, however were also associated with moderate-severe haemorrhage and pneumothorax in $7.5 \%$ and $7.7 \%$, respectively [18]. 


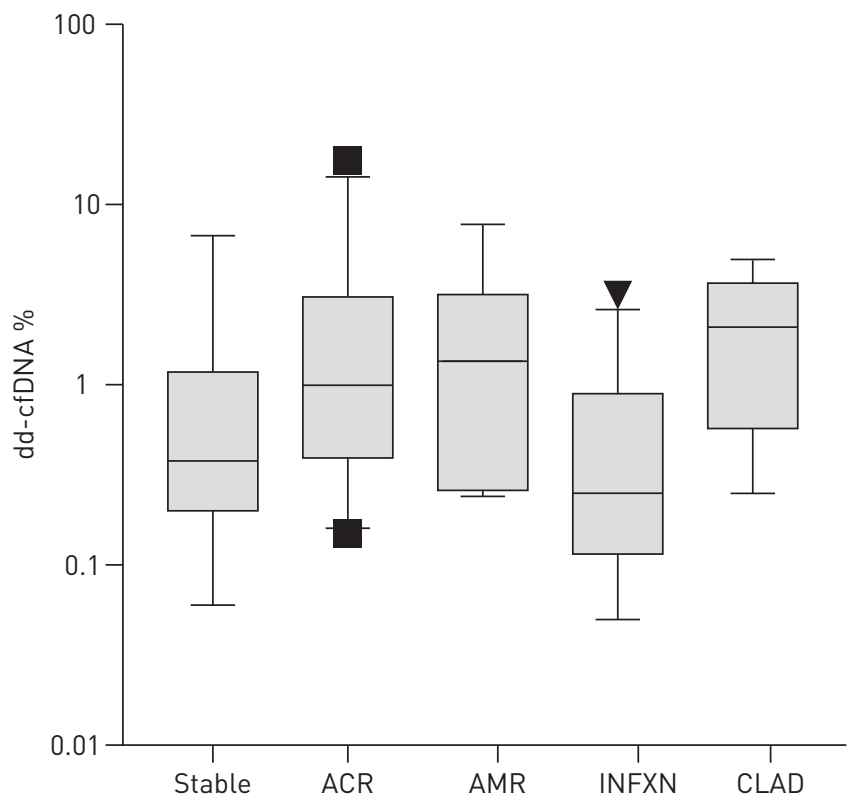

FIGURE 2 Box plots representing median and 25-75th quartiles (interquartile range) for donor-derived cell-free DNA (dd-cfDNA) levels ( $\log _{10} y$-axis) associated with ( $x$-axis) stable healthy, acute cellular rejection $(A C R)$, antibody-mediated rejection (AMR), chronic lung allograft dysfunction-obstructive phenotype (CLAD) and infection in absence of concurrent rejection (INFXN). Wilcoxon rank sum tests for cohorts with ACR ( $p=0.02)$, AMR ( $p=0.07)$, bronchiolitis obliterans syndrome (BOS) $(p=0.02)$ and INFXN ( $p=0.56)$ compared with the stable cohort.

Therefore, implementation of dd-cfDNA as a plasma biomarker of allograft "tissue injury" [18]. Therefore, a clinically validated plasma biomarker of rejection with "tissue injury" should be valuable as a tool for lung transplantation clinical care and surveillance.

Limitations to our study included the use of archived biorepository plasma samples; however, these were associated with appropriate clinical-pathological data to allow assignment to the different cohort categories. Admittedly further clarification of the INFXN cohort with additional radiographic and clinical data would have allowed for a more detailed analysis. We speculate that limitations in sample size likely accounted for a lack of statistical significance for the observed elevation in dd-cfDNA with AMR.

FIGURE 3 Individual donor-derived cell-free DNA (dd-cfDNA) levels (y-axis) for unique lung transplant patients associated with the cohorts ( $x$-axis) of healthy stable, acute cellular rejection (ACR), antibody-mediated rejection (AMR), allograft infection in the absence of associated rejection (INFXN) and obstructive chronic lung allograft dysfunction (CLAD). Horizontal line depicts cohort median value.

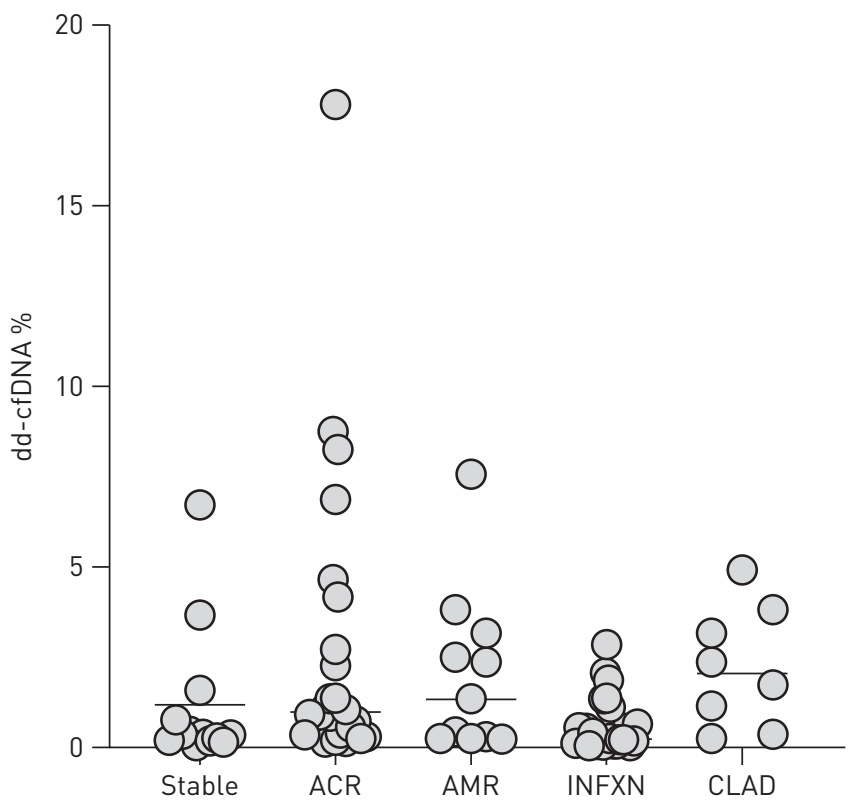




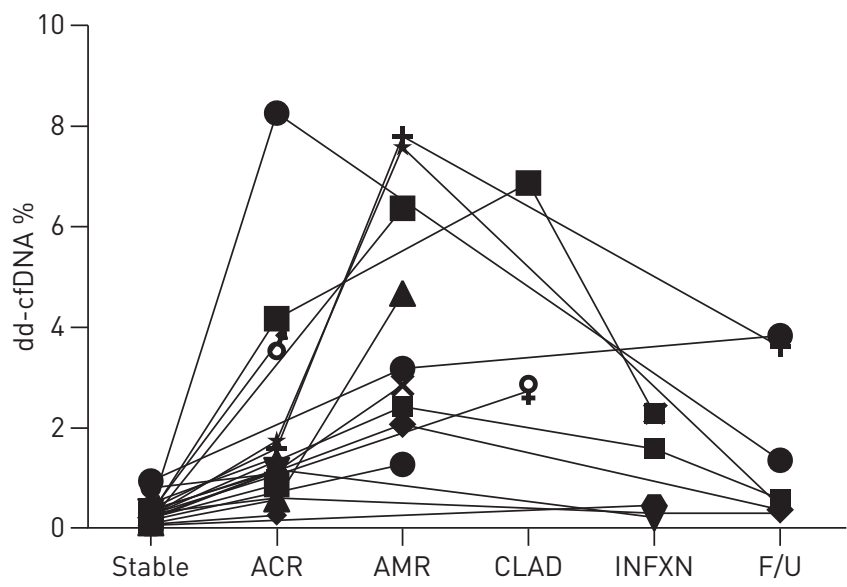

FIGURE 4 The different donor-derived cell-free DNA (dd-cfDNA) levels ( $y$-axis) across distinct clinical events ( $x$-axis) for 19 unique patients with multiple associated plasma samples. Clinical events included: healthy stable, acute cellular rejection (ACR), antibody-mediated rejection (AMR), chronic lung allograft dysfunction (CLAD), allograft infection in absence of concurrent rejection (INFXN) and follow-up (F/U) testing 1-2 months after treatment. Individual patient dd-cfDNA levels demonstrated a trend for higher levels in association with AMR and CLAD clinical events.

Nevertheless, there was an evident trend (1.34\%; IQR: 0.34-2.40) for this cohort which should be further assessed in the context of a prospective multi-centre study. Additionally, a preliminary estimate of a threshold dd-cfDNA level for aggregated allograft rejection was determined from this data; however, this threshold will require further prospective validation. During our analysis, we included ISHLT Grade A1 ("minimal") ACR episodes, despite less certainty regarding clinical significance of these episodes. Intriguing was the observation that despite only minimal perivascular lymphocytic infiltration, the elevated dd-cfDNA levels with Grade A1 ACR were not statistically different from the values with Grades A2-A4, which suggested a similar extent of tissue injury occurred during the Grade A1 rejection events. Indeed, GLANVILLE et al. [19] had previously reported an increased predisposition to BOS (68\% versus 43\%) in lung transplantation recipients with multiple episodes of Grade A1 ACR compared to those who experienced $\leqslant 1$ episode. Additionally, $\sim 25 \%$ of Grade A1 episodes progressed to high-grade ACR within the subsequent 3-month period [19]. Therefore, histopathological diagnosis alone may lack adequate sensitivity for determining the biological effect of ACR on allograft injury that may require specific therapeutic interventions. We speculate that the histopathological diagnosis of ACR may be further complemented by dd-cfDNA assessment of allograft injury. Another potential limitation to our study is that we did not stratify episodes of Grade A1-A4 ACR for the presence or absence of associated lymphocytic bronchiolitis (Grade B 0-2R), and we specifically excluded specimens with an isolated lymphocytic bronchiolitis from the healthy NORMAL cohort to avoid the potential confounding variable. As previously reported by Ross et al. [20], isolated lymphocytic bronchiolitis is likely to be the incipient histopathological lesion in the continuum of obstructive CLAD. Therefore, assessment of dd-cfDNA in isolated lymphocytic bronchiolitis will be a critical area of further investigation, to determine associated allograft tissue injury and thereby evaluate subtle patterns of ACR that may require treatment as a pre-emptive strategy to mitigate development of CLAD or BOS.

The analytics of the NGS targeted sequencing dd-cfDNA measurement conformed to strict quality control standards in a CLIA and American CAP-certified laboratory, and plasma samples were of adequate requisite volume for analysis. This plasma dd-cfDNA assay (AlloSure; CareDx, Inc.) interrogates the disparity for a targeted panel of single nucleotide polymorphisms across all 22 somatic chromosomes to determine the donor fraction (\%) and does not require genomic sequencing of donor or recipient [14]. Importantly, a rapid turnaround time from phlebotomy to reported result of $<72 \mathrm{~h}$ in the USA for this test would permit implementation into lung transplantation surveillance protocols for clinical utility.

\section{Conclusions}

These data suggest clinical utility for measurement by NGT sequencing of dd-cfDNA as a plasma biomarker of "tissue injury" in the spectrum of allograft rejection events after unilateral and bilateral lung transplantation. An "optimal threshold" value for dd-cfDNA from our investigation was $0.85 \%$; however, this would require further clinical prospective validation. Further, a specific threshold value for dd-cfDNA may be less relevant than the monitoring of longitudinal trends during surveillance for maintenance of 
allograft health [13]. Future studies would appear warranted to evaluate this plasma biomarker of "allograft injury" in the context of HLA- and non-HLA-associated AMR, and isolated lymphocytic bronchiolitis in the continuum of obstructive CLAD. Further prospective studies incorporating dd-cfDNA as a noninvasive tool for detection of rejection and assessment of lung allograft health may prove valuable.

Author contributions: Study design and implementation by all authors. Statistical analysis performed by K.K. Khush and D.J. Ross. Biorepository clinical-pathologic data obtained by H. Luikart. Initial manuscript draft jointly by K.K. Khush and D.J. Ross. Manuscript edits, revisions and final draft performed and approved by all authors.

Conflict of interest: K.K. Khush reports grants and personal fees from CareDx, Inc., outside the submitted work. I. De Vlaminck has nothing to disclose. H. Luikart has nothing to disclose. D.J. Ross has nothing to disclose. M.R Nicolls has nothing to disclose.

\section{References}

1 Chambers DC, Cherikh WS, Harhay MO, et al. The International Thoracic Organ Transplant Registry of the International Society for Heart and Lung Transplantation: Thirty-sixth adult lung and heart-lung transplantation Report-2019; Focus theme: Donor and recipient size match. J Heart Lung Transplant 2019; 38: 1042-1055.

2 Kulkarni HS, Cherikh WS, Chambers DC, et al. Bronchiolitis obliterans syndrome-free survival after lung transplantation: An International Society for Heart and Lung Transplantation Thoracic Transplant Registry analysis. J Heart Lung Transplant 2019; 38: 5-16.

3 Bloom RD, Bromberg JS, Poggio ED, et al. Cell-free DNA and active rejection in kidney allografts. J Am Soc Nephrol 2017; 28: 2221-2232.

4 Jordan SC, Bunnapradist S, Bromberg JS, et al. Donor-derived cell-free DNA identifies antibody-mediated rejection in donor specific antibody positive kidney transplant recipients. Transplant Direct 2018; 4: e379.

5 De Vlaminck I, Valantine HA, Snyder TM, et al. Circulating cell-free DNA enables noninvasive diagnosis of heart transplant rejection. Sci Transl Med 2014; 6: 241 ra77.

6 Agbor-Enoh S, Wang Y, Tunc I, et al. Donor-derived cell-free DNA predicts allograft failure and mortality after lung transplantation. EBioMedicine 2019; 40: 541-553.

7 Agbor-Enoh S, Jackson AM, Tunc I, et al. Late manifestation of alloantibody-associated injury and clinical pulmonary antibody-mediated rejection: evidence from cell-free DNA analysis. J Heart Lung Transplant 2018; 37: 925-932.

8 Stewart S, Fishbein MC, Snell GI, et al. Revision of the 1996 working formulation for the standardization of nomenclature in the diagnosis of lung rejection. J Heart Lung Transplant 2007; 26: 1229-1242.

9 Wallace WD, Weigt SS, Farver CF. Update on pathology of antibody-mediated rejection in the lung allograft Curr Opin Organ Transplant 2014; 19: 303-308.

10 Levine DJ, Glanville AR, Aboyoun C, et al. Antibody-mediated rejection of the lung: a consensus report of the International Society for Heart and Lung Transplantation. J Heart Lung Transplant 2016; 35: 397-406.

11 Verleden GM, Raghu G, Meyer KC, et al. A new classification system for chronic lung allograft dysfunction. J Heart Lung Transplant 2014; 33: 127-133.

12 Glanville AR, Verleden GM, Todd JL, et al. Chronic lung allograft dysfunction: definition and update of restrictive allograft syndrome - a consensus report from the Pulmonary Council of the ISHLT. J Heart Lung Transplant 2019; 38: 483-492.

13 Bromberg JS, Brennan DC, Poggio E, et al. Biological variation of donor-derived cell-free DNA in renal transplant recipients. J Appl Lab Med 2017; 2: 309-321.

14 Grskovic M, Hiller DJ, Eubank LA, et al. Validation of a clinical-grade assay to measure donor-derived cell-free DNA in solid organ transplant recipients. J Mol Diagn 2016; 18: 890-902.

15 Khush KK, Patel J, Pinney S, et al. Noninvasive detection of graft injury after heart transplant using donor-derived cell-free DNA: a prospective multicenter study. Am J Transplant 2019; 19: 2889-2899.

16 Trulock EP, Ettinger NA, Brunt EM, et al. The role of transbronchial lung biopsy in the treatment of lung transplant recipients. An analysis of 200 consecutive procedures. Chest 1992; 102: 1049-1054.

17 Vitulo P, Cremaschi P, Arbustini E, et al. Surveillance transbronchial biopsy in the diagnosis of acute lung rejection in heart and lung and lung transplant recipients. Monaldi Arch Chest Dis 1996; 51: 12-15.

18 Loor K, Culebras M, Sansano I, et al. Optimization of transbronchial cryobiopsy in lung transplant recipients. Ann Thorac Surg 2019; 108: 1052-1058.

19 Hopkins PM, Aboyoun CL, Chhajed PN, et al. Association of minimal rejection in lung transplant recipients with obliterative bronchiolitis. Am J Respir Crit Care Med 2004; 170: 1022-1026.

20 Ross DJ, Marchevsky A, Kramer M, et al. 'Refractoriness' of airflow obstruction associated with isolated lymphocytic bronchiolitis/bronchitis in pulmonary allografts. J Heart Lung Transplant 1997; 16: 832-838. 